Pacific Journal of Mathematics

A NOTE ON THE GROUP STRUCTURE OF UNIT REGULAR 


\title{
A NOTE ON THE GROUP STRUCTURE OF UNIT REGULAR RING ELEMENTS
}

\author{
Robert E. HARTwig AND JiANG LUH
}

\begin{abstract}
Local properties of unit regular ring elements are investigated. It is shown that an element of a ring $R$ with unity is regular if and only if there exists a unit $u \in R$ and a group $G$ such that $a \in u G$.
\end{abstract}

1. Introduction. It is well-known that $[15,7]$ a ring $R$ is strongly regular if and only if every $a \in R$ is a group member. In this note we shall use the basic theorem for group members in a ring to show locally that a ring element $a \in R$ (with unity) is unit regular exactly when there is a unit $u \in R$ and a group $G$ in $R$ such that $a \in u G$. Hence unit regular rings are, as it were locally a "rotated" version of strongly regular rings.

We remind the reader that a ring $R$ is called regular if for every $a \in R, a \in a R a$; strongly regular if for every $a \in R, a \in a^{2} R$, and unit regular if for every $a \in R$, there is a unit $u \in R$ such that $a u \alpha=$ $a$ [3]. Similar definitions hold locally. A ring with unity is called finite if $a b=1$ implies $b a=1$. Any solution $a^{-}$to $a x a=a$ is called an inner or 1-inverse of [1], while any solution $a^{+}$to $a x a=a$ and $x a x=x$ is called a reflexive or 1-2 inverse of $a$.

For idempotents $e$ and $f$ in $R, e \sim f$ denotes the equivalence in the sense of Kaplansky [13] as contrasted with $a \stackrel{u}{\sim} b$ which denotes that $a=p b q$ with $p$ and $q$ invertible.

As usual, similarity will be denoted by $\approx$, the right and left annihilators of $a \in R$ will be denoted by $a^{0}=\{x \in R: a x=0\},{ }^{0} a=$ $\{x \in R: x a=0\}$ respectively, while interior direct sums and isomorphisms are denoted by + and $\cong$ respectively. A ring $R$ is called faithful if $a R=(0)$ implies $a=0$.

We shall make use of the following fundamental theorem for group members.

THEOREM 1. Let $S$ be a semigroup and $a \in S$. The following are equivalent.

1. a is a group member.

2. $a$ has a group inverse $a^{\sharp}$ in $S$ which satisfies $a x a=a, x a x=$ $x$ and $a x=x a$.

3. $a$ has a commutative inner inverse $a^{-}$which satisfies $a x a=$ $a$, and $a x=x a$.

4. $a S=e S, S a=S e$ and $a \in e S e$ for some idempotent $e \in S$.

5. $a \in a^{2} S \cap S a^{2}$. 
6. $a \in \alpha^{-} a S a \alpha^{=}$for some inner inverses $a^{-}, a^{=}$in $S$.

7. $a S=a^{+} S$ for some reflexive inverse $a^{+}$in $S$.

7a. $S a=S a^{+}$for some reflexive inverse $a^{+}$in $S$.

8. $a S=a^{-} a S$ for some inner inverse $a^{-}$in $S$.

8a. $S a=S a a^{-}$for some inner inverse $a^{-}$in $S$.

If in addition $S=R$ is a faithful ring, these are equivalent to

9. $R=a R+a^{0}$.

9a. $R=R a+{ }^{0} a$.

In any of the above cases $a^{\#}$ and $e=a a^{\#}$ are unique and the maximal subgroup containing a is given by

$$
\begin{aligned}
H_{a} & =\left\{x \in S: x^{\sharp} \text { exists, } x x^{\#}=a a^{\sharp}=e\right\} \\
& =\{x \in S: x S=a S, S x=S a, x \in a S a\} .
\end{aligned}
$$

Proof. For a proof of the equivalence of (1)-(5); we refer to $[14,7,8]$.

$(1) \Rightarrow(6): \quad$ Clearly, $a=a^{\#} a^{3} a^{\#}$.

$(6) \Rightarrow(7)$ : Let $a=a^{-} a z \alpha a^{=}$for some $z \in S$ and set $a^{+}=a^{-} a a^{=}$. Then $\alpha=\alpha^{-} \alpha \alpha^{=} \alpha z \alpha \alpha^{=}=\alpha^{+} \alpha z \alpha \alpha^{=} \in \alpha^{+} S$.

On the other hand, since $a^{3}=\alpha\left(\alpha^{-} \alpha z \alpha \alpha^{=}\right) \alpha=\alpha z \alpha$, we have $a=$ $a^{-} a^{3} a^{=}$, and $a^{3} \alpha^{=}=a^{2}=\alpha^{-} a^{3}$. Hence $a^{+}=a^{-} \alpha \alpha^{=}=a^{-}\left(a^{-} a^{3} a^{=}\right) \alpha^{=}=$ $a^{-} a^{2} a^{=} a^{=}=a^{-} a^{3}\left(\alpha^{=}\right)^{3}=\alpha\left(\alpha^{=}\right)^{2} \in \alpha S$, and so $\alpha^{+} S=\alpha S$.

$(7) \Longrightarrow(8)$ : Obvious, since $\alpha^{+} S=a^{+} \alpha S$.

$(8) \Rightarrow(1)$ : If $a S=a^{-} a S$, then $a^{2}=a^{-} a x$ for some $x$. Hence $a^{-} a a^{2}=a^{2}$ or $a^{-} a^{3}=a^{2}$.

Similarly, $a^{-} a=a y$ for some $y$, and so $a=a^{2} y$. By a result of Drazin [2] the index of $\alpha$ equals one and $a^{\#}$ exists.

The results $7 \mathrm{a}$ and $8 \mathrm{a}$ follow by symmetry.

We remark that an element $a \in R$ for which $a R=a^{+} R$ or $R \alpha=$ $R \alpha^{+}$for some $a^{+}$, generalizes so called $E P$ elements [16, 7, 1] for which $a R=a^{-} R=a^{\dagger} R, R *$-regular, where $a^{\dagger}$ is the Moore-Penrose inverse of $\alpha$. Thus in a *-regular ring, an $E P$ element belongs to some group $G$.

For a proof of $(9) \Longrightarrow(1)$ for the case where $R$ has a unity 1 or is regular, we refer to [7]. When $R$ is faithful we have to proceed as follows. $R=a R+a^{0} \Longrightarrow \alpha=a r+n, \alpha n=0 \Longrightarrow \alpha=a(\alpha s+m)+n$, for some $s \in R, m \in a^{0}$. Hence $a^{2}=a^{4} b$, for some $b \in a R$. Also $a(a x)=$ $0 \Longrightarrow a x \in a R \cap a^{0}=(0)$, so that $\left(a^{2}\right)^{0}=a^{0}$. Hence $R=a^{2} R+\left(a^{2}\right)^{0}$. It then follows that $b=\left(a^{2}\right)^{\sharp}$, since

$$
a^{2}\left(a^{2}-a^{2} b a^{2}\right)=a^{2}\left(a^{2} b-b a^{2}\right)=a^{2}\left(b a^{2} b-b\right)=0 \text {. }
$$

Because $a^{2}$ commutes with $a$, it follows by a result of Drazin [2] that $\left(\alpha^{2}\right)^{\sharp} \alpha=a\left(\alpha^{2}\right)^{\sharp}$. Now $\left(\alpha-\alpha^{2}\left(\alpha^{2}\right)^{\sharp} \alpha\right) R=\left(\alpha-a^{2}\left(\alpha^{2}\right)^{\sharp} \alpha\right) \alpha R=(0)$ and 
hence if $R$ is faithful, $a=a^{2}\left(a^{2}\right)^{\sharp} a=a^{2}\left(a\left(a^{2}\right)^{\sharp}\right)$. One may now repeat the above argument to show that $a^{\#}=a\left(a^{2}\right)^{\#}=a^{\sharp} a a^{\sharp}$.

That $(1) \Rightarrow(9)$ is clear.

Before giving our main result several remarks should be made here.

REMARK 1. The condition "faithful" may be replaced by the weaker condition

$$
\text { for every } \quad r \in R \quad r^{0} \cap{ }^{0} R=(0) \text {. }
$$

This may not be dropped entirely as seen from the example

$$
R=\left\{\left[\begin{array}{cc}
0 & \alpha \\
0 & 0
\end{array}\right]: \alpha \text { is a real number }\right\}, \alpha=\left[\begin{array}{ll}
0 & 1 \\
0 & 0
\end{array}\right], a R=(0), \alpha^{0}=R
$$

and ${ }^{\circ} R=R$. Here $R=a R+a^{0}$, yet $a^{\#}$ clearly does not exist since $a^{2}=0$.

REMARK 2. For a regular ring $R$ with unity, (1.1) may be written as [10]

$$
H_{a}=\{x \in R: x=p a=a q \text { for some units } p \text { and } q\} .
$$

REMARK 3. If $a$ has a unique reflexive inverse $a^{+}$then $a^{\sharp}$ exists, and if $a$ has a unique idempotent of the form $a a^{+}$then $a \in a^{2} R$. Hence if either of them hold globally, then $R$ is stronly regular. These results are easy consequences of the fact that the class $\left\{a^{+}\right\}$of all reflexive inverses of $a$ is given by [9],

$$
\left[a^{+}+\left(1-a^{+} a\right) R\right] \alpha\left[a^{+}+R\left(1-a a^{+}\right)\right] .
$$

2. Main results. We begin with several preliminary results which will be used in our main theorem.

LEMMA 1. If $R$ is a ring with unity 1 , and if $\phi: a R \rightarrow b R$ is $a$ module isomorphism, where $a$ and $p=\phi(a)$ are regular elements, then $R a=R p$ and $p R=b R$.

Proof. $\phi(a)=\phi\left(a a^{-} a\right)=\phi\left(a a^{-}\right) a$ and $\phi(\alpha)=p p^{-} p \Rightarrow a=\phi^{-1}\left(p p^{-}\right) p=$ $\phi^{-1}\left(p p^{-}\right) \phi(a)$. The following is given in [10].

Lemma 2. If $a$ and $b$ are regular elements in a ring $R$ with unity 1 , then

$$
a R=b R \quad \text { and } \quad R a=R b \Longleftrightarrow b=u a=a v
$$


for some units $u, v$ in $R$.

LEMMA 3. Let $R$ be a ring with unity 1 and $a$ and $b$ be regular elements in $R$. Then the following are equivalent:

(i ) $b \stackrel{u}{\sim} a$;

(ii) $a a^{-} \approx b b^{-}$and $a^{-} a \approx b^{-} b$, for some, and hence all $a^{-}, b^{-}$;

(iii) $a a^{-} \sim b b^{-}, 1-a a^{-} \sim 1-b b^{-}$, and $1-a^{-} a \sim 1-b^{-} b$, for some and hence all $a^{-}, b^{-}$;

(iv) $a R \cong b R$ and $R / a R \cong R / b R, R / R a \cong R / R b$.

Proof. (i) $\Rightarrow$ (ii): If $b=p a q$, for some units $p$ and $q$, then for any particular $a^{-}, q^{-1} a^{-} p^{-1} \in\left\{b^{-}\right\}$, and hence $p a a^{-} p^{-1} \in\left\{b b^{-}\right\}, q^{-1} a^{-} a q \in$ $\left\{b^{-} b\right\}$. Now for any $a^{=} \in\left\{a^{-}\right\}, b^{=} \in\left\{b^{-}\right\}, a a^{=} \sim a a^{-}, b b^{=} \sim b b^{-}$and thus $a a^{=} \approx a a^{-} \approx p a a^{-1} p^{-1} \approx b b^{=}$.

(ii) $\Rightarrow$ (i): Let $a a^{-}=u b b^{-} u^{-1}, a^{-} a=v^{-1} b^{-} b v$. Then $a R=u b v R$, $R a=R u b v$. Lemma 2 now ensures that $a=u b v p=q u b v$ for some units $p, q$ and thus $a \stackrel{u}{\sim} b$.

The equivalence of (ii) and (iii) is well-known since $a a^{-} \approx b b^{-} \Leftrightarrow$ $a a^{-} \sim b b^{-}$and $1-a a^{-} \sim 1-b b^{-}$, while $a a^{-} \sim b b^{-} \Leftrightarrow a^{-} a \sim b^{-} b$, [11].

(i) $\Rightarrow$ (iv): If $b=p a q$ where $p$ and $q$ are units, then $a R \cong b R$ and $1-b b^{-}=p\left(1-a a^{-}\right) p^{-1} \Rightarrow\left(1-b b^{-}\right) R=p\left(1-a a^{-}\right) R \cong\left(1-a a^{-}\right) R$. Lastly, since $b R+\left(1-b b^{-}\right) R=R=a R+\left(1-a a^{-}\right) R \Rightarrow R / a R \cong$ $\left(1-a a^{-}\right) R$ and $R / b R \cong\left(1-b b^{-}\right) R$, the results follows.

(iv) $\Rightarrow$ (ii): If $a R \cong b R$ and $R / a R \cong R / b R$, then $\left(1-a a^{-}\right) R \cong$ $R / a R \cong R / b R \cong\left(1-b b^{-}\right) R$ and so $a a^{-} \sim b b^{-}, 1-a a^{-} \sim 1-b b^{-}$. It follows that $a a^{-} \approx b b^{-}$. Similarly, $a^{-} a \approx b^{-} b$.

We note in passing that the statement $R / a R \cong R / b R$ is clearly equivalent to the statement " $a R$ and $b R$ have all direct summands isomorphic."

Lemma 4. If $a \in R$ is a regular element of $R$ and $1 \in R$, then for all units $u, v \in R,\left\{(u a v)^{-}\right\}=v^{-1}\left\{a^{-}\right\} u^{-1}$.

Proof. This is an easy consequence of the fact that the class of all inner inverses of $b$ is given by $\left\{b^{-}\right\}=b^{-}+\left(1-b^{-} b\right) R+R\left(1-b b^{-}\right)$.

We now come to the main theorem of this paper, which gives numerous conditions for a ring element to be unit regular.

THeOREM 2A. Let $R$ be a ring with unity 1 and let $a \in R$. Then the following are equivalent:

1. $a u a=a$ for some unit $u$ in $R$. 
2. $(a u)^{\#}$ exists for some unit $u$ in $R$.

2a. $(u a)^{\#}$ exists for some unit $u$ in $R$.

3. au has a commutative inner inverse for some unit $u$ in $R$.

3a. ua has a commutative inner inverse for some unit $u$ in $R$.

4. $a u R=e R$ and $R a u=R e$ for some unit $u$ and idempotent $e$ in $R$.

4a. $u a R=e R$ and $R u a=R e$ for some unit $u$ and idempotent $e$ in $R$.

5. $a \in \alpha u a R \cap R a u a$ for some unit $u$ in $R$.

6. $R=a R+u\left(a^{0}\right)$ for some unit $u$ in $R$.

6a. $R=R a+\left({ }^{0} a\right) u$ for some unit $u$ in $R$.

Proof. $\quad(1) \Longrightarrow(2)$ : Clearly, $a u \alpha=\alpha \Longrightarrow(\alpha u)^{2}=a u \Longrightarrow(\alpha u)^{\sharp}$ exists.

$(2) \Longrightarrow(1)$ : Observe that $\alpha u\left[(\alpha u)^{\#}+\left(1-(\alpha u)^{\sharp} \alpha u\right)\right] \alpha u=a u \Rightarrow a u v a=$ $a$, where $v=(\alpha u)^{\#}+\left(1-(\alpha u)^{\sharp} \alpha u\right)$ and $v^{-1}=\alpha u+1-(\alpha u)^{\sharp} \alpha u$.

$(2) \Leftrightarrow(2 \mathrm{a}): \quad u \alpha=u(\alpha u) u^{-1}$ and so $(u \alpha)^{\#}$ exists exactly when $(\alpha u)^{\#}$ exists.

Since idempotents clearly are group members, it is obvious that $a$ is unit regular precisely when $a \in u G$ for some group $G$ and unit $u$ in $R$. The equivalence of (2) through (6a) follows immediately from Theorem 1, applied to the group members $a u$, and $u \alpha$. For example, $a u \in(\alpha u)^{2} R \cap R(\alpha u)^{2} \Leftrightarrow \alpha \in \alpha u \alpha R \cap R \alpha u \alpha$ and $(u \alpha)^{\#}$ exists $\Leftrightarrow R=$ $u a R+(u a)^{0} \Leftrightarrow R=a R+u^{-1}\left(\alpha^{0}\right)$. If we are given in addition that $a \in R$ is a regular element, then several important additional conditions may be given for $a$ to be unit regular.

THEOREM 2B. If $R$ is a ring with unity 1 and $a \in R$ is a regular element, then the following are equivalent to a being unit regular.

(7) $a \in u^{-1} \alpha^{-} a R a \alpha^{=} u^{-1}$ for some unit $u$ and some inner inverses $a^{-}, \alpha^{=}$in $R$.

(8) $a^{-} x a=y, a y a^{=}=x$, where $a^{-}, a^{=}$are inner inverses of $a \Rightarrow$ $x \approx y$.

(9) $c a=a c, c \in R \Rightarrow c a \alpha^{=} \approx a^{-}$ac for some and hence all inner inverses $a^{-}, a^{=}$in $R$.

(10) $a a^{-} \approx a^{-} a$ for some and hence all inner inverses $a^{-}$in $R$.

(11) $a R=u \alpha^{-} a R$ for some unit $u$ and some inner inverse $a^{-}$ in $R$.

(12) $a R=u a^{+} R$ for some unit $u$ and some reflexive inverse $a^{+}$ in $R$.

(13) $a R=e R$, with $e^{2}=e \Longrightarrow a u=e$ for some unit $u$ in $R$.

(14) $a R=b R$ with $b$ unit regular $\Longrightarrow a g=b$ for some unit $g$ in $R$.

(15) $a R \stackrel{\phi}{\cong} b R$, with $\phi(a), b$ unit regular $\Longrightarrow \alpha \stackrel{u}{\sim} b$.

(16) $a R \stackrel{\phi}{\cong} b R$, with $\phi(a)$, bunit regular $\Rightarrow R / a R \cong R / b R$, 
together with their left analogues.

Proof. $\quad(2) \Leftrightarrow(7)$ : By Theorem 1(6), $(\alpha u)^{\#}$

exists $\Longleftrightarrow a u \in(a u)^{-} a u R a u(a u)^{=} \Longleftrightarrow a u \in u^{-1} a^{-} a R a a^{=}$

$\Longleftrightarrow a \in u^{-1} a^{-} a R a a^{=} u^{-1}$,

for some inner inverses $a^{-}, a^{=}$of $a$. It should be noted that Lemma 4 was also used.

(1) $\Rightarrow(8)$ : Let $a u a=a$, where $u$ is a unit. Then $y=a^{-} x a=$ $a^{-} a y a^{=} a \Rightarrow y=y \alpha^{=} a=a^{-} a y=y \alpha^{-} a$, and $x=a y \alpha^{=}=\alpha \alpha^{-} x a a^{=} \Rightarrow a \alpha^{=} x=$ $x=a a^{-} x=x a a^{=}$. Also, clearly, $a y=x a$ and $y u \alpha=y \alpha^{=} a u \alpha=y \alpha^{=} a=$ $y, a u x=x$. Now note that $y=a^{-} a y \approx u a y$ since $u a y\left(1-a^{-} a+u a\right)=$ $u a y=\left(1-a^{-} a+u a\right) a^{-} a y$ and so, $y=a^{-} a y \approx u a y=u x a=u(x a u) u^{-1}$. Next, again $x u \alpha \approx x a \alpha^{=}=x$, for

$$
\left(1-a \alpha^{=}+a u\right) x a u=x \alpha u=x \alpha \alpha^{=}\left(1-a \alpha^{=}+\alpha u\right) .
$$

And so, $y=q^{-1} x q$, where $q=\left(1-a a^{=}+a u\right) u^{-1}\left(1-a^{-} a+u a\right)$.

$(8) \Rightarrow(9)$ : Since $\alpha^{-}\left(c a a^{=}\right) \alpha=\alpha^{-} a c$ and $\alpha\left(a^{-} a c\right) \alpha^{=}=c a \alpha^{=}$, the result follows at once from (9).

$(9) \Rightarrow(10):$ Because $a a^{-} \approx a a^{=}$for any $a^{-}, a^{=}$, we simply set $c=1$ in $(9)$.

$(10) \Rightarrow(11): \quad a \alpha^{-} \approx a^{-} a \Rightarrow a a^{-}=u \alpha^{-} a u^{-1}$ for some unit $u \Rightarrow a R=$ $u a^{-} a R$ as desired.

$(11) \Rightarrow(12): \quad a R=u a^{-} a a^{-} a R=u a^{+} a R=u a^{+} R$, where $a^{+}=a^{-} a a^{-}$.

$(12) \Rightarrow(2 \mathrm{a})$ : Let $a R=u a^{+} R$. Then $u^{-1} a R=a^{+} R=a^{+} u R=$ $\left(u^{-1} a\right)^{+} R$, and hence by Theorem $1(7),\left(u^{-1} a\right)^{\sharp}$ exists.

$(1) \Rightarrow(13):$ If $a R=e R$ and $a u a=\alpha, u$ unit, $e^{2}=e$, then $a u R=$ $e R \Rightarrow a u e=e$. Hence $a u v=e$, where $v=1-a u+e, v^{-1}=1+a u-e$. Thus $\alpha$ and $e$ are right associates.

$(13) \Rightarrow(14)$ : If $a R=b R, b v b=b$ and $v$ is a unit, then $a R=e R$, where $e=b v$. By (13), $a u=e=b v$ for some unit $e$. Hence $a u v^{-1}=b$ as desired.

$(14) \Rightarrow(1)$ : Since $a R=a a^{-} R$, and $a \alpha^{-}$is unit regular, (14) implies that $a g=a a^{-}$for some unit $g$. Hence $a g a=a$ as requested. It is now clear by symmetry, that the left analogues of the above results also are equivalent to element $a$ being unit regular.

$(14) \Rightarrow(15)$ : Suppose that (14) and hence its left analogue (14a) both hold.

Now let $a R \cong \phi(a) R=b R$ and $p=\phi(a)$. Then by Lemma 1 , $R a=R p$ and $p R=b R$, so that by (14) and (14a), $p v=b$ and $u a=p$ for some units $u$ and $v$. These are in fact given by $u=\left(p^{=}\right)^{-1}\left(1+p^{=} p-a^{=} a\right) a^{=}, v=p^{=}\left(1-p p^{=}+b b^{=}\right)\left(b^{=}\right)^{-1}$, in which $a^{=}$, $b^{=}$, and $p^{=}$are unit inner inverses. Hence $b=u a v$, as desired. 
$(15) \Rightarrow(16)$ : This follows immediately from Lemma 3 .

$(16) \Longrightarrow(1)$ : Since $a R \stackrel{\phi}{\cong} a^{-} a R$, where $\phi(a)=a^{-} a=b$, it follows that $a a^{-} \sim b, 1-a a^{-} \sim 1-b$, so that $a a^{-} \approx b$.

Hence, by Lemma $3, u a v=b=a^{-} a$ for some units $u, v$, which implies that $u a v u a v=u a v$ or $a(v u) a=a$, as desired. Alternatively, (10) could be used.

The remaining results follow again by symmetry.

REMARK 1. In (8), we proved the conjecture made in [12] that pseudosimilarity implies similarity in a unit regular ring. Pseudosimilarity, $\bar{\sim}$, is defined by

Definition 1. $x \approx y$ if $a^{-} x a=y, a y a^{=}=x$ for some $a$ and its inner inverses $a^{-}, a^{=}$.

REMARK 2. The equivalence of (1) and (6) was also proved by Ehrlich [4] who used endomorphism rings. As shown above it is actually a simple consequence of the fundamental Theorem 1 .

REMARK 3. Part (10) should be compared with the global result of Vidav [17] and Fuchs [5], which state that a regular ring $R$ is unit regular exactly when $e^{2}=e \sim f=f^{2} \Rightarrow e \approx f$ [17] or when $a R \cong$ $b R \Longrightarrow R / a R \cong R / b R[5]$.

REMARK 4. The global analogue of (16) is that a regular ring $R$ is unit regular exactly when $a R \cong b R$ implies that $a R$ and $b R$ have a common direct summand [6].

One final remark is here needed, namely, if $R$ is a unit regular ring and if $\phi: a R \rightarrow b R$ is $a n y$ isomorphism, then, by Lemma $1, R a=$ $R \phi(a)$ and hence by (14a) $\phi(a)=u a$ for some unit $u$.

We have thus shown:

COROLlaRY 1. In a unit regular ring $R$, all right module isomorphisms $\phi: a R \rightarrow b R$, are of the form $\phi(a r)=u a r$, where $u$ is $a$ unit. Similarly, all left module isomorphisms $\phi: R a \rightarrow R b$ are of the form $\phi(r a)=r a v$, for some unit $v \in R$.

The converse of these statements always hold.

3. The unit inner inverses. We shall now examine more closely the class $\mathscr{U}_{a}$ of unit inner inverses of a given element $a$ of a unit regular ring. 
We begin by noting that if $a u \alpha=a$, with $u$ invertible then $\mathscr{C}_{a}$ can be represented as

$$
\mathscr{U}_{a}=u \mathscr{U}_{a u}=\mathscr{Q}_{u a} u \text {. }
$$

Indeed, if $w \in \mathscr{Q}_{a u}$, then $a u w a u=a u$ which implies that $a u w a=$ $a$ and hence $u w \in \mathscr{L}_{a}$, while conversely, if $a w a=a, w$ a unit, then $a u\left(u^{-1} w\right) a u=a u$ which implies that $u^{-1} w \in \mathscr{U}_{a u}$ and hence $w \in u \mathscr{U}_{a u}$. The second identity follows similarly.

Since $u \mathscr{U}_{a u}$ is independent of the choice of the unit inner inverse $u$ of $a$, we have, for any unit inner inverses $u$ and $v$ of $a$,

$$
\mathscr{U}_{a}=u \mathscr{U}_{a u}=v \mathscr{U}_{a v},
$$

so that in particular, $u^{-1} v \in \mathscr{U}_{a u}$.

Consequently, the set $\mathscr{U}_{a}$ is determined by the set of unit inner inverses $\mathscr{U}_{e}$ of the idempotent element $e=a u$. When $e^{2}=e$, there are several representations for $\mathscr{U}_{e}$. In fact, $\mathscr{C}_{e}$ is the set of all units of the form:

$$
\begin{array}{ll}
\text { (i ) } 1+(1-e) x+y(1-e) & \text { for some } x, y \text {; } \\
\text { (ii) } e+(1-e) v+s(1-e) & \text { for fome } v, s \text {; } \\
\text { (iii) } 1+h-e h e & \text { for some } h \text {; } \\
\text { (iv) } e+k-e k e & \text { for some } k .
\end{array}
$$

In general, the set $\mathscr{U}_{a}$ or even $\mathscr{W}_{e}$ will not be a union of semigroups. For example, if $e=\left[\begin{array}{ll}1 & 0 \\ 1 & 0\end{array}\right] \in \boldsymbol{R}_{2 \times 2}$, where $\boldsymbol{R}_{2 \times 2}$ denotes the two by two matrix ring over the real field, then it is easy to see that $\left[\begin{array}{rr}-1 & 2 \\ 0 & 2\end{array}\right] \in \mathscr{U}_{e}$, but $\left[\begin{array}{rr}-1 & 2 \\ 0 & 2\end{array}\right]^{2} \notin \mathscr{U}_{e}$.

In fact, it is only for idempotent elements possible to possess union of semigroups of unit inner inverses.

Proposition 3. Let a be a unit regular element of a ring $R$ with unity 1.

(i) If the set $\mathscr{L}_{a}$ of unit inner inverses of $a$ is a union of semigroups then $a^{2}=a$.

(ii) If $R$ is a prime ring and if $\mathscr{C}_{a}$ forms a semigroup, then $a=0$ or $a=1$.

Proof. (i) Let $a u a=a$ with $u$ a unit. Then $u^{2} \in \mathscr{U}_{a}$ and $a u^{2} a=a$. Now consider: $a u(1+a u(1-a u)) a=a u a+a u(1-a u) a=a+a-$ $a=a$, which implies that $u(1+a(1-a u)) \in \mathscr{U}_{a}$. Thus $(u(1-a(1-$ $a u)))^{2} \in \mathscr{L}_{a}$. That is, $a=a(u(1-a(1-a u)))^{2} a=(a u-a(1-a u)) u(1-$ $a(1-a u)) \alpha=\left(a u^{2}-a u+a^{2} u^{2}\right)\left(a-a^{2}+a^{2}\right)=a u^{2} a-a u a+a^{2} u^{2} a=$ $a-a+a^{2}=a^{2}$.

(ii) Now suppose that $a=e=e^{2}$. Then clearly $1+e R(1-e)$ and $1+(1-e) R e$ are contained in $\mathscr{U}_{e}$. Hence by the semigroup 
assumption, $e(1+e R(1-e))(1+(1-e) R e) e=e$ which implies that

$$
e R(1-e) R e=0 \text {. }
$$

Since $R$ is prime, it follows that either $e=0$ or $e=1$ as desired.

REMARK 1. In (ii), the primeness cannot be dropped as seen from the example of semiprime ring $R=Z_{2} \oplus Z_{2}$, where $Z_{2}$ denotes the Galois field of order 2. Here $\left.\mathscr{U}_{(1,0)}\right)=\mathscr{U}_{(0,1)}=\{(1,1)\}$ is a semigroup, yet $(1,0)$ and $(0,1)$ are neither zero element nor unity element.

REMARK 2. The same conclusions may be drawn if the element is just regular and the set $\left\{a^{-}\right\}$of inner inverses forms a semigroup. In fact, if $a b a=a$ then $a b^{2} a=a$ and also $a\left(b-b a+b a^{2} b\right) a=a \Rightarrow$ $a\left(b-b a+b a^{2} b\right) b a=a \Rightarrow a=a-a b a+a^{2} b^{2} a=a^{2}$.

The rest follows as in part (ii).

REMARK 3. For an invertible element $1+h$-ehe in a unit regular ring, ehe need not lie in $H_{e}$. For example, if $e=\left[\begin{array}{ll}1 & 0 \\ 1 & 0\end{array}\right] \epsilon$ $\boldsymbol{R}_{2 \times 2}$ and $h=\left[\begin{array}{ll}0 & 0 \\ 1 & 0\end{array}\right]$, then $1+h-$ ehe $=\left[\begin{array}{ll}1 & 0 \\ 1 & 1\end{array}\right]$ is invertible but ehe $=$ $\left[\begin{array}{ll}0 & 0 \\ 0 & 0\end{array}\right] \notin H_{e}$.

There are five sets of units that appear naturally in the study of $\mathscr{U}_{e}$. These are:

1. $P_{e}=1+(1-e) R e=\left\{u \in \mathscr{Q}_{e}: e(1-u)(1-e)=0\right\}$,

2. $Q_{e}=1+e R(1-e)=\left\{u \in \mathscr{U}_{e}:(1-e)(1-u) e=0\right\}$,

3. $V_{e}=\left\{v \in \mathscr{U}_{e}: e v=e\right\}$,

4. $W_{e}=\left\{w \in \mathscr{Q}_{e}: w e=e\right\}$, and

5. $C_{e}=\{z \in R: e z=z e, z$ is a unit $\}$.

For example, $1-a a^{=}+a a^{\equiv} \in W_{a a^{-}}$for any inner inverses $a^{-}, a^{=}, a^{\equiv}$ of $a$.

It is easily seen that

(i) all these sets are semigroups (in fact monoids).

(ii) $P_{e} \subseteq V_{e} \subseteq \mathscr{U}_{e}, Q_{e} \subseteq W_{e} \subseteq \mathscr{U}_{e}, V_{e} \cap W_{e}=\{1+(1-e) x(1-e) \epsilon$ $\left.\mathscr{U}_{e}: x \in R\right\}$.

(iii) $P_{e} \cap Q_{e} \subseteq V_{e} \cap W_{e}=\mathscr{U}_{e} \cap C_{e} \subseteq C_{e}$.

In addition it is known that [14]

(iv) $e C_{e}=H_{e}$ is the maximal subgroup containing $e$. Moreover, it is easily shown that

(v) $V_{e} \mathscr{U}_{e} W_{e}=\mathscr{U}_{e}=P_{e} \mathscr{U}_{e} Q_{e}$, for let $u \in \mathscr{L}_{e}, v \in V_{e}, w \in W_{e}$, then evuwe $=e u e=e$, while conversely $u=1 \cdot u \cdot 1$ ensures the first equality. The second equality follows similarly. 
It should be remarked here that in general $P_{e} \neq V_{e}, Q_{e} \neq W_{e}$, for again let $e=\left[\begin{array}{ll}0 & 0 \\ 0 & 1\end{array}\right]$ and $x=\left[\begin{array}{ll}x_{1} & x_{3} \\ x_{2} & x_{4}\end{array}\right]$ in $\boldsymbol{R}_{2 \times 2}$ with $x_{1} \neq 0$ and $1+x_{1}$ invertible. Then $1+(1-e) x=\left[\begin{array}{cc}1+x_{1} & x_{3} \\ 0 & 1\end{array}\right] \in V_{e}$, while $\left[\begin{array}{cc}x_{1} & x_{3} \\ 0 & 0\end{array}\right] \neq y e$ for any $y \in \boldsymbol{R}_{2 \times 2}$.

Before examining the subgroup $H_{e}$, let us first prove a global conjecture made in [11]. We start with

Lemma 4. Let $R$ be a ring with unity 1 . Then the following two conditions are equivalent.

(i) $R$ is unit regular such that every nonzero element in $R$ has a unique inner inverse;

(ii) $R$ contains only idempotent elements and invertible elements.

Proof. (i) $\Rightarrow$ (ii): Suppose $a^{2} \neq a \in R$ and $a u a=a, u$ a unit $u \neq 1$. Then

$$
\alpha u(1-a(1-a u)) \alpha=\alpha=a(1-(1-u \alpha) \alpha) u \alpha
$$

where $(1-a(1-a u))^{-1}=1+a(1-a u)$ and

$$
(1-(1-u a) a)^{-1}=1+(1-u a) a .
$$

Hence by uniqueness, $u(1-a(1-a u))=u=(1-(1-u a) a) u$ or $a(1-a u)=0=(1-u a) a$. Now $a^{2} u=a=u a^{2}$ implies by Theorem 1 , that $a$ has a group inverse $a^{\#}=u a u$. Consequently, $a u=a a^{\#}=$ $a^{\sharp} a=u a$. Since $a\left(a^{\sharp}+1-a a^{\sharp}\right) \alpha=a$ and $\left(a^{\sharp}+1-a a^{\sharp}\right)^{-1}=a+1-a \alpha^{\sharp}$, it follows by uniqueness that $u=u a u+1-a u$ or $u(1-a u)=1-$ $a u$. Multiplying this by $1-a u$, we obtain

$$
(1-a u) u(1-a u)=1-a u \text {. }
$$

Now either $1-a u=0$ or $1-a u \neq 0$. Since $1-a u \neq 0$ is idempotent and $(1-a u) 1(1-a u)=1-a u$, uniqueness implies that $u=1$, which is impossible. Hence $a u=1=u \alpha$ and $a$ is a unit.

(ii) $\Rightarrow$ (i): It is clear that $R$ is a regular ring. Now let $a \in R$ and $a \neq 0$. First suppose $a=1$. Then $a u a=a$ implies that $u=1$ and so is unique. Next, suppose $a \neq 1$. If $a^{2}=a$ and $a u a=a$, where $u$ is a unit $\neq 1$, then $1-u$ is also a unit. For otherwise $(1-u)^{2}=1-u$ would imply that $u^{2}=u$ which forces $u$ to equal 1 . Now, since $a$ is not a unit, $a(1-u)$ is not a unit. Hence $[a(1-u)]^{2}=$ $a(1-u)$. This implies that $a=a(1-u) a=a^{2}-a u a=a^{2}-a=0$, a contradiction. Hence $u=1$ and the unit inner inverse of $a$ is unique. If $a$ is a not idempotent then $a$ is a unit and clearly $a^{-1}$ is the only unit inner inverse of $a$, completing the proof. 
We may now sharpen this to the following.

THEOREM 4. Let $R$ be a unit regular ring. If every nonzero element of $R$ has a unique unit inner inverse then either $R$ is a Boolean ring or $R$ is a division ring.

Proof. Suppose $R$ is neither Boolean nor a division ring. Then there exists $a \in R$ such that $a^{2} \neq a$ and there are $x \neq 0, y \neq 0$ in $R$ such that $x y=0$, (since it is well-known that a regular integral domain must be a field). By Lemma $4, a$ is a unit and $x$ and $y$ are idempotents. Now, consider element $a x$. If $(a x)^{2}=a x$ then

$$
a(x a-1) x=0 \Longrightarrow(x a-1) x=0 \Longrightarrow x=x a x \Longrightarrow a=1,
$$

by the uniqueness of unit inner inverses of $x$. This yields a contradiction. On the other hand, if $(a x)^{2} \neq a x$ then $a x$ must be a unit which implies that $x$ is a unit and thus that $y=0$, which again is a contradiction. Thus $R$ must be either a division ring or a Boolean ring.

Let us now consider briefly the maximal subgroup

$$
H_{e}=\{x \in R: x R=e R, R x=R e\}
$$

which contains the idempotent element $e \in R$. We begin with a global result.

Proposition 5. If $R$ is a regular ring with unity 1 and $e$ is an idempotent element in $R$, then

$$
H_{e}=\{e u e: \text { eueve }=e=e v e u e, u, v \text { units in } R\} \text {. }
$$

This says that the e-units in eRe are all of the form eue for some 1-unit $u \in R$.

Proof. It is well-known that

$$
\begin{aligned}
H_{e} & =\{\text { ere }: \text { erese }=e=\text { esere } ; r, s \in R\} \\
& =\{\text { ere }: \text { ere } R=e R, \text { Rere }=R e\} .
\end{aligned}
$$

By Lemma 3, for ere $\in H_{e}$ there are units $u, v$ in $R$ such that ereu $=e=$ vere, which implies that $($ ere $)(e u e)=e=($ eve $)($ ere $)$. The uniqueness of $e$-inverses ensures that $e u e=e v e$.

Now again by Lemma 3, since eueR $=e R$ and Reue $=R e$, there are units $w, z$ in $R$, such that euew $=e=z e u e$. Consequently, euewe= $e=e z e u e$. And so, by uniqueness, ewe $=e z e=e r e$. Hence we may replace in each element ere the element $r$ by a 1-unit $w \in R$. 
Conversely, it is easily seen that this set is contained in $H_{e}$.

We remark that when $R$ is a finite regular ring [11] we may shorten this to

$$
H_{e}=\{\text { eue: eueve }=e ; u, v \text { units in } R\} .
$$

Suppose now again that $a u \alpha=\alpha=\alpha v a$, with $u, v$ units in $R$. Then if we set $e=a u, f=a v$, we have $a \in H_{a u} u^{-1}$, and more generally, $a \in \bigcap\left\{H_{a u} u^{-1}: u \in \mathscr{U}_{a}\right\}$. Since $e R=f R=a R$, it follows that $e f=f, f e=e$ and that $e \approx f$. In fact, if $w=1-e+f=(1+e-f)^{-1}=$ $1-a(u-v)$, then $e w=w f=f$ and thus

$$
w H_{f} w^{-1}=H_{e},
$$

that is, the subgroups $H_{a u}$ and $H_{a v}$ are isomorphic. It follows similarly that

$$
H_{u a}=u H_{a u} u^{-1},
$$

because $x \in H_{u a} \Leftrightarrow u^{-1} x u \in H_{a u}$. And so, the subgroups $H_{a u}, H_{u a}, H_{a v}$, $H_{v a}$ are all isomorphic.

4. Conclusions. We have seen that an element $a \in R$ is unit regular exactly when $a \in u G$ for some unit $u$ and group $G$ in $R$. In the same way that the concept of a Drazin inverse $a^{d}$ (see $\left.[1,2]\right)$ generalizes that of a group inverse $a^{\sharp}$ to the case that $\left(a^{k}\right)^{\sharp}$ exists for some $k \geqq 1$, we may generalize the concept of a unit regular element.

Definition 2. (i) An element $a \in R$ is $k$-unit regular if $a^{k}$ is unit regular for some $k \geqq 1$.

(ii) An element $a \in R$ is unit-Drazin invertible if there is a unit $u \in R$ such that $(u a)^{k}$ is a group member for some $k \geqq 1$.

By Theorem 2, the former is equivalent to $R=a^{k} R+u\left(a^{k}\right)^{0}$, while the latter reduces to the existence of $(u a)^{d}$.

In closing we mention of few open problems relating to $\mathscr{U}_{a}$ in a unit regular ring. Let $e$ be an idempotent element.

1. For what $h$ is $1+h$-ehe invertible?

2. For what $x$ is $1+(1-e) x$ invertible?

3. How are $\mathscr{C}_{e}$ and $H_{e}$ related?

4. What sort of subgroup is $\cap\left\{H_{a u}: u \in \mathscr{Q}_{a}\right\}$ ?

5. For what type of regular semigroups does Theorem 2,1-2 remain valid? 


\section{REFERENCES}

1. A. Ben Israel and T. N. E. Greville, Generalized Inverse Theory and Applications, Wiley, New York, 1974.

2. M. P. Drazin, Pseudo-inverses in associative rings and semigroups, Amer. Math. Monthly, 65 (1958), 506-514.

3. G. Ehrlich, Unit regular rings, Portugal Math., 27 (1969), 209-212.

4. - Units and one-sided units in regular rings, Trans. Amer. Math. Soc., 216 (1976), 81-90.

5. L. Fuchs, On the substitution property for modules, Monatsh. Math., (1971), 198-204.

6. D. Handelman, Perspectivity and cancellation in regular rings, to appear.

7. R. E. Hartwig, Block generalized 'inverses, Arch. Rath. Mech. Anal., 61 (1976), $187-251$.

8. - More on the Souriau-frame Algorithm and the Drazin inverse, SIAM J. Appl. Math., 31 (1976), 42-46.

9. - 1-2 Inverses and the invariance of $B A^{+} C$, Linear Algebra Appl., 11 (1975), $271-275$.

10. - Generalized inverses, Ep Elements and Associates, to appear, in Revue Roumaine.

11. R. E. Hartwig and Jiang Luh, On finite regular rings, Pacific J. Math., 69 (1977), 73-95.

12. R. E. Hartwig and F. Hall, Pseudo-similarity for matrices over fields, submitted for publication.

13. I. Kaplansky, Rings of Operators, W. A. Benjamin Inc., New York, 1968.

14. G. L. Losey and H. Schneider, Group membership in rings and semigroups, Pacific J. Math., 11 (1961), 1089-1098.

15. Jiang Luh, A note on strongly regular rings, Proc, Japan Acad., 40 (1964), 74-75.

16. H. Schwerdtfeger, Introduction to Linear Algebra and the Theory of Matrices, 2nd Ed., P. Noordhoff, Grominger, 1962.

17. I. Vidav, Modules over regular rings, Mathematica Balkamica, 1 (1971), 287-292.

Received August 17, 1976 and in revised form March 3, 1977.

UNIVERSITY OF GRAZ

Graz, Austria 8010

AND

North Carolina State University

RALEIGH, NC 27607 



\section{PACIFIC JOURNAL OF MATHEMATICS}

\section{EDITORS}

RICHARD ARENS (Managing Editor)

University of California

Los Angeles, CA 90024

Charles W. Curtis

University of Oregon

Eugene, OR 97403

C. C. MOORE

University of California

Berkeley, CA 94720

\section{J. DugundJI}

Department of Mathematics

University of Southern California

Los Angeles, CA 90007

R. FinN and J. Milgram

Stanford University

Stanford, CA 94305

ASSOCIATE EDITORS
E. F. BECKENBACH
B. H. NeumanN
F. WOLF
K. YOSHIDA

\section{SUPPORTING INSTITUTIONS}

UNIVERSITY OF BRITISH COLUMBIA CALIFORNIA INSTITUTE OF TECHNOLOGY

UNIVERSITY OF CALIFORNIA

MONTANA STATE UNIVERSITY

UNIVERSITY OF NEVADA, RENO

NEW MEXICO STATE UNIVERSITY

OREGON STATE UNIVERSITY

UNIVERSITY OF OREGON

OSAKA UNIVERSITY

\author{
UNIVERSITY OF SOUTHERN CALIFORNIA \\ STANFORD UNIVERSITY \\ UNIVERSITY OF HAWAII \\ UNIVERSITY OF TOKYO \\ UNIVERSITY OF UTAH \\ WASHINGTON STATE UNIVERSITY \\ UNIVERSITY OF WASHINGTON \\ AMERICAN MATHEMATICAL SOCIETY
}

The Supporting Institutions listed above contribute to the cost of publication of this Journal, but they are not owners or publishers and have no responsibility for its content or policies.

Mathematical papers intended for publication in the Pacific Jaurnal of Mathematics should be in typed form or offset-reproduced, (not dittoed), double spaced with large margins. Please do not use built up fractions in the text of your manuscript. You may however, use them in the displayed equations. Underline Greek letters in red, German in green, and script in blue. The first paragraph or two must be capable of being used separately as a synopsis of the entire paper. Items of the bibliography should not be cited there unless absolutely necessary, in which case they must be identified by author and Journal, rather than by item number. Manuscripts, in triplicate, may be sent to any one of the editors. Please classify according to the scheme of Math. Reviews, Index to Vol. 39. All other communications should be addressed to the managing editor, or Elaine Barth, University of California, Los Angeles, California, 90024.

The Pacific Journal of Mathematics expects the author's institution to pay page charges, and reserves the right to delay publication for nonpayment of charges in case of financial emergency.

100 reprints are provided free for each article, only if page charges have been substantially paid. Additional copies may be obtained at cost in multiples of 50 .

The Pacific Journal of Mathematics is issued monthly as of January 1966. Regular subscription rate: $\$ 7200$ a year (6 Vols., 12 issues). Special rate: $\$ 36.00$ a year to individual members of supporting institutions.

Subscriptions, orders for back numbers, and changes of address should be sent to Pacific Journal of Mathematics, 103 Highland Boulevard, Berkeley, California, 94708.

PUBLISHED BY PACIFIC JOURNAL OF MATHEMATICS, A NON-PROFIT CORPORATION

Printed at Kokusai Bunken Insatsusha (International Academic Printing Co., Ltd.). 8-8, 3-chome, Takadanobaba, Shinjuku-ku, Tokyo 160, Japan.

Copyright (C) 1975 by Pacific Journal of Mathematics Manufactured and first issued in Japan 


\section{Pacific Journal of Mathematics}

\section{Vol. 71, No. $2 \quad$ December, 1977}

Krishnaswami Alladi and Paul Erdős, On an additive arithmetic

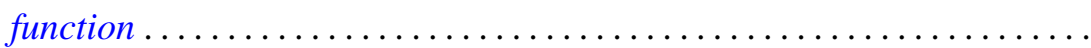

James Bailey and Dale Rolfsen, An unexpected surgery construction of a

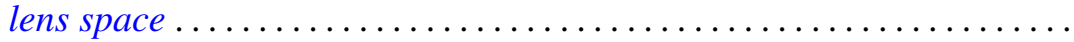

Lawrence James Brenton, On the Riemann-Roch equation for singular

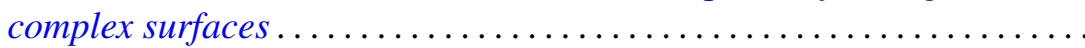

James Glenn Brookshear, Projective ideals in rings of continuous

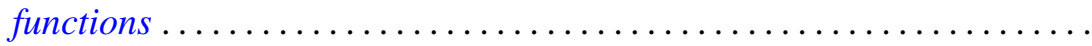

Lawrence Gerald Brown, Stable isomorphism of hereditary subalgebras of

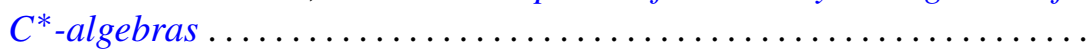

Lawrence Gerald Brown, Philip Palmer Green and Marc Aristide Rieffel, Stable isomorphism and strong Morita equivalence of $C^{*}$-algebras....

N. Burgoyne, Robert L. Griess, Jr. and Richard Lyons, Maximal subgroups

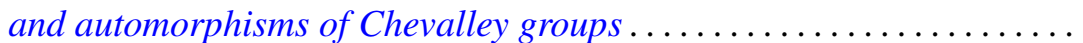
365

Yuen-Kwok Chan, Constructive foundations of potential theory .... 405

Peter Fletcher and William Lindgren, On $w \Delta$-spaces, $w \sigma$-spaces and

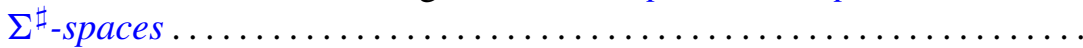

Louis M. Friedler and Dix Hayes Pettey, Inverse limits and mappings of

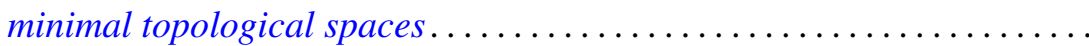

Robert E. Hartwig and Jiang Luh, A note on the group structure of unit regular ring elements.

I. Martin (Irving) Isaacs, Real representations of groups with a single involution ...

Nicolas P. Jewell, The existence of discontinuous module derivations . .

Antonio M. Lopez, The maximal right quotient semigroup of a strong semilattice of semigroups .......................

Dennis McGavran, $T^{n}$-actions on simply connected $(n+2)$-manifolds

Charles Anthony Micchelli and Allan Pinkus, Total positivity and the exact $n$-width of certain sets in $L^{1}$.

Barada K. Ray and Billy E. Rhoades, Fixed point-theorems for mappings with a contractive iterate .......................

Fred Richman and Elbert A. Walker, Ext in pre-Abelian categories. .

Raymond Craig Roan, Weak* generators of $H^{\infty}$ and $l^{1}$..

Saburou Saitoh, The exact Bergman kernel and the kernels of Szegö type...

Kung-Wei Yang, Operators invertible modulo the weakly compact 